\title{
The ALOHA Trial: (Intra-Articular Local Anaesthetic in Hip Arthroscopy) - A Double-Blinded, 3-Arm Randomised Controlled Clinical Trial Comparing Pre-Emptive, High- and Low-Dose Intra-Articular Local Anaesthetic in Hip Arthroscopy
}

Chong Oon Tan ( $\nabla$ drchongtan@gmail.com )

Austin Health https://orcid.org/0000-0003-2873-5976

Phong Tran

Western Health

YewMing Chong

Western Health

William Howard

Austin Health

Laurence Weinberg

Austin Health

Research article

Keywords: Local Anaesthesia; Hip Arthroscopy; Acute Pain; Fascia lliaca Block

Posted Date: July 25th, 2019

DOl: https://doi.org/10.21203/rs.2.11094/v1

License: (a) This work is licensed under a Creative Commons Attribution 4.0 International License.

Read Full License 


\section{Abstract}

Background Pain after hip arthroscopy is variable but can be severe [11-point Numerical Rating Scale (NRS-11) pain scores of 10] despite multimodal analgesia. Although postoperative rescue regional analgesia is useful in these cases its efficacy may be limited by the maximum safe dose of local anaesthetic (LA) permissible when high-dose intra-articular local anaesthetic (IALA) has already been used. IALA may reduce acute postoperative pain after hip arthroscopy, however neither its optimum dose nor timing of administration have been systematically evaluated. Methods In 132 randomly assigned adult patients scheduled for therapeutic hip arthroscopy we compared the effects of two different doses of IALA given at procedure end (Group L [low-dose]: 100mg ropivacaine; Group H [high-dose]: $200 \mathrm{mg}$ ropivacaine). We also investigated the effect of an additional pre-emptive dose at the beginning of the procedure (Group P [pre-emptive]: 100mg ropivacaine at procedure start and end). Results There were no statistically significant differences between groups for NRS-11 pain scores in recovery (mean[SD]: Group $L$ - 2.2[1.9]; Group H - 2.3[2.1]; Group P - 2.7[2.5]; lowest $p=0.6$ ), or post recovery Visual Analogue Scale (VAS) pain scores [largest mean difference VAS 1.5 hours: $5 \mathrm{~mm}(p=0.32)$; VAS 2 hours: $5 \mathrm{~mm}(p=$ $0.35)$; VAS 4 hours: $2 \mathrm{~mm}(p=0.7)$; VAS 6 hours: $3 \mathrm{~mm}(p=0.7)]$. There were also no significant differences in antiemetic usage and requirement for rescue fascia iliaca block (FIB) between groups. Conclusions Compared to a single $100 \mathrm{mg}$ dose of ropivacaine at the end of the procedure, we were unable to demonstrate any advantage of either a higher dose IALA or a pre-emptive dose IALA when multimodal analgesia is used. Lower-dose IALA could reduce total systemic LA absorption if a given rescue regional analgesic LA dose is used postoperatively.

\section{Background}

Acute pain at rest following hip arthroscopy varies greatly but can be severe (11-point Numerical Rating Scale [NRS] up to 10[1]) despite multimodal systemic analgesic therapy, requiring total intraoperative and recovery room IV morphine doses of up to $0.3 \mathrm{mg} / \mathrm{kg}$ IV morphine[1]. Pre-emptive regional analgesic techniques offer superior analgesia but carry with them significant risks of falls[2], delayed mobilization[3] and neuropraxias[4]. Fascia iliaca blockade (FIB) has been shown to be a useful analgesic adjunct[5] but in our practice has only been used as a rescue analgesic in approximately 3-4\% of cases[1]. The precise cause of severe early postoperative pain at rest after hip arthroscopy is unknown; use of high pressure intra-articular fluid infusion has been implicated[1], suggesting that the degree of peri-articular soft tissue swelling may be causative. It is a frequent clinical observation that after approximately 6 hours soft tissue swelling improves and coincides with rapid improvement in pain and mobility. This rapid improvement continues to the morning of postoperative day 1 when almost all patients are able to be discharged home on crutches with minimal oral analgesia.

Intra-articular local anaesthetic (IALA) for hip arthroscopy has been shown to be of analgesic benefit in some small studies[6, 7]. Local anaesthetic (LA) agents have been shown to confer pre-emptive analgesic 
effects[8], however no comparative literature exists regarding the optimal dose and timing of IALA administration. If maximum recommended IALA doses are more efficacious than lower doses, side effects of systemic analgesics and the need for rescue regional analgesia may be reduced; conversely, if lower dose IALA is similarly efficacious, a given FIB rescue dose will be less likely to result in higher systemic LA absorption. Hence we hypothesized that the pre-emptive as well as postoperative administration of, and increased postoperative dosing of IALA, would have improved analgesic efficacy and postoperative opioid usage in patients undergoing therapeutic hip arthroscopy.

\section{Methods}

This study was approved by our institution's Human Research and Ethics committee (Melbourne Health HREC, approval \# 2013.195) and registered under the Australian \& New Zealand Clinical Trials Register (ACTRN \#12614001121651). Approval was granted for protocol variation when increased sample size was necessary to avoid a Type 2 error in the outcome measures (\#2013.195.P1-2). We conducted this study as per CONSORT recommendations. (See figure 1.) All patients provided written informed consent to be included in the trial. Inclusion criteria were age $>18$ years, and planned therapeutic hip arthroscopy for procedures on the ligamentum teres, labrum, or articular aspects of the femur or acetabulum. Patients were excluded if the arthroscopic procedure planned was for ilio-tibial band release alone, or if there were contraindications to any of the perioperative drugs in the study protocol. The primary study outcome measures were median recovery 11-point Numerical Rating Scale (NRS-11) assessments of pain, and Visual Analogue Scale (VAS) assessments of pain measured at 1.5, 2, 4 and 6 hours postoperatively at rest. Secondary outcome measures included highest and lowest recovery NRS-11 at rest, recovery analgesic and anti-emetic usage, total $\& 24$ hour postoperative opioid analgesic and anti-emetic use, and repeated analysis of all pain outcomes corrected for pre-operative assessments of pain (ie. study VAS pre-operative VAS = corrected VAS). Patients performed their preoperative VAS assessment at rest in the supine position whilst in the pre-anaesthesia bay. Recovery NRS-11 assessments were made 5-10 minutely depending on frequency of titrated IV morphine bolus requirements, until the patient's NRS-11 was $<5$ or until analgesia was defined as satisfactory by the patient, at which time they were discharged to the ward. Postoperative VAS time points for pain assessment and the 24 hour postoperative period were calculated from the time of application of surgical traction. Ward VAS assessments were performed by nursing staff, instructing patients to mark a single line across an uninterrupted horizontal scale where the extreme left- and right-hand extremes of the scale were marked as "No Pain" with smiling face schematic, and "Pain as Bad as it Possibly Could Be" with crying face schematic, respectively.

132 adult patients were recruited from a single surgeon's practice and randomly allocated to 1 of 3 groups: i) Low-dose LA (Group L - 100mg ropivacaine at end of procedure), ii) High-dose LA (Group H $200 \mathrm{mg}$ ropivacaine at end of procedure) and iii) Pre-emptive LA (Group P - 100mg ropivacaine just prior to and at end of procedure). Random permuted block sizes of 9, 12 and 15 were used to achieve balanced group sizes whilst minimizing excessive runs of single group allocations. A web-based 
randomization program (Sealed Envelope Ltd, London, UK) was used by a team member not involved with assessment of any patient outcome measures to allocate each patient's treatment group. After randomization subjects' IALA/ saline syringe orders were then allocated in a sealed envelope prior to the day of surgery. On the day of surgery the scrub scout nurse prepared IALA/ placebo syringes in the preparation room, separate from the operating room and out of sight from other team members, and did not share knowledge of syringe contents. Syringes were prepared under sterile conditions and as per the opened envelope instructions: Group L - pre-emptive syringe $20 \mathrm{ml}$ normal saline/ postoperative syringe $20 \mathrm{ml} 0.5 \%$ ropivacaine; Group $\mathrm{H}$ - pre-emptive syringe $20 \mathrm{ml}$ normal saline/ postoperative syringe $20 \mathrm{ml}$ $1 \%$ ropivacaine; Group P - pre-emptive syringe $20 \mathrm{ml} 0.5 \%$ ropivacaine/ postoperative syringe $20 \mathrm{ml} 0.5 \%$ ropivacaine. Pain assessments were performed by the recovery room nursing staff and postoperative ward staff, all of whom were blinded to the patients' group allocation.

Intraoperative anaesthesia and analgesia was standardized to spontaneous ventilation sevoflurane/ oxygen: air [50:50\%] laryngeal mask anaesthesia, induction with 1-2mg/ kg IV propofol and $10-15 \mathrm{mcg} /$ $\mathrm{kg}$ IV alfentanil, and administration of $1 \mathrm{mg} / \mathrm{kg}$ IV tramadol, $0.05 \mathrm{mcg} / \mathrm{kg}$ IV morphine, $40 \mathrm{mg}$ IV parecoxib and $1 \mathrm{~g} \mathrm{IV} \mathrm{paracetomol} \mathrm{from} \mathrm{the} \mathrm{start} \mathrm{of} \mathrm{the} \mathrm{procedure.} \mathrm{All} \mathrm{patients} \mathrm{received} 8 \mathrm{mg}$ dexamethasone and $1 \mathrm{mg}$ granisetron IV as preventative anti-emesis. Hip arthroscopy was performed in the lateral decubitus position and surgical traction was applied with a McCarthy Hip Distractor (Innomed, Inc, Savannah, GA, USA). Intra-articular access was facilitated with an arthroscopic infusion of Ringer's Lactate at constant pressures of $40 \mathrm{mmHg}$. Ligamentum Teres tears were debrided, and synovitis treated with synovectomy, with the aid of a radiofrequency ablation probe (Vulcan Eflex Ablator Probe, Smith \& Nephew, Andover, MA, USA). Labral tears were treated with circumferential suture anchor refixation (Nanotack Flex, Stryker, Sunnyvale, CA USA).

Pre-emptive LA was administered as soon as the initial arthroscopy portal was inserted into the hip joint, whilst LA administered at the end of the procedure was given via the arthroscopic portal just before portal removal. The study recovery protocol for postoperative analgesia offered subjects 1 ) IV morphine $1 \mathrm{mg}$ (if NRS-11 <7) to $2 \mathrm{mg}$ (if NRS-11 7 or above) $5-10$ minutely, titrated up to $0.2 \mathrm{mg} / \mathrm{kg}$ for clinical review or until dose limiting side effects such as sedation or nausea and vomiting occurred, simultaneously with 2) IV tramadol 50mg 15 minutely, up to a total theatre and recovery room dose not exceeding $200 \mathrm{mg}$. Breakthrough antiemetics offered were cyclizine $50 \mathrm{mg}$ IV (1 dose) and droperidol $10 \mathrm{mcg} / \mathrm{kg}$ ( $1 \mathrm{dose})$.

Patients were offered rescue FIB if recovery NRS-11 pain assessments were $>5$ despite maximum study protocol systemic analgesia, or if sedation, nausea or vomiting became rate-limiting factors to further administration of IV morphine. FIB was performed as originally described by Dalens[9] with $20 \mathrm{ml}$ of $0.5 \%$ ropivacaine. Patients with an NRS persisting above 5 or who were still dissatisfied with their analgesia at 
this point were offered IV morphine via a Patient Controlled Analgesia device (PCA) and ketamine infusion of $0.1-0.2 \mathrm{mg} / \mathrm{kg} / \mathrm{hr}$, and were included in the intention-to-treat analysis. Once discharged to the ward patients were initially offered $2.5-5 \mathrm{mg}$ oral oxycodone as first-line analgesia, $50-100 \mathrm{mg}$ oral tramadol as second-line analgesia, and $2.5-5 \mathrm{mg}$ intramuscular or subcutaneous morphine as third-line analgesia every 6 hours. $1 \mathrm{mg}$ IV bd granisetron and $50 \mathrm{mg}$ IV tds cyclizine were offered as needed for nausea and vomiting. Opioid doses were expressed as IV morphine equivalents; for tramadol we used the manufacturer's recommended equipotency of $1 \mathrm{mg} I V$ morphine $=10 \mathrm{mg}$ IV tramadol; for oxycodone, $2 \mathrm{mg}$ oral oxycodone $=1 \mathrm{mg} \mathrm{IV} \mathrm{morphine[10].}$

\section{Statistical Analysis}

All calculations were performed using GraphPad Prism 5 (GraphPad Software, CA, USA) and G*Power 3.1 (University of Dusseldorf, Dusseldorf, Germany) statistical software. As the difference between median and mean of each dataset was $<25 \%$ of the lower value and sample sizes $>30$ we used parametric analyses. Categorical data were compared with chi-squared testing, and continuous data analysed with one-way ANOVA and Bonferroni multiple comparisons testing, with Bonferroni correction of $p$-values. (Recovery NRS-11: highest, median and lowest NRS-11 values; Postoperative VAS: mean values at 1.5, 2, 4 and 6 hours) between multiple pairs of groups. Patients lost to follow up with missing VAS data had all other trial data included in the final analysis; missing data was not imputed.

\section{Power calculation}

Sample size was determined "a-priori" with the use of G*Power V 3.1 (University of Dusseldorf, Dusseldorf, Germany). Morganthaler et al found an overall reduction in VAS at rest after hip arthroscopy of [mean (SD)] of $10 \mathrm{~mm}(18 \mathrm{~mm})$ over a 20 -hr postoperative period when comparing the effect of IA Bupivucaine $50 \mathrm{mg}$ against placebo. Assuming approximate LA potency of $1 \mathrm{mg}$ Bupivucaine $=1.5-2 \mathrm{mg}$ Ropivacaine, and a linear dose-response of relationship between LA dose and reduction in postoperative VAS, each $100 \mathrm{mg}$ of IALA Ropivacaine reduces postoperative VAS by [mean (SD)] $10 \mathrm{~mm}(18 \mathrm{~mm})$. With desired power of 0.8 the number of patients per arm required to demonstrate a mean VAS difference of $10 \mathrm{~mm}$ by 2-tailed unpaired t-test at alpha of 0.05 was 17 patients. We chose to consider a $10 \mathrm{~mm}$ VAS difference as significant because the use of multimodal analgesia would most likely reduce measured differences in the outcomes. Sample sizes were more than doubled in our study to account for multiple comparisons. The low frequency of need for rescue FIB in this population would require large sample sizes to detect differences between groups and hence we did not power this study for that purpose.

\section{Results}


Consistent with our study protocol of strict intention-to-treat analysis, all patients with missing postoperative pain score data had other retained data included in the final analysis. There were no acute complications from the use of IALA or rescue FIB. All patients were discharged home on the morning of postoperative day 1 with the use of crutches and oral analgesia. Figure 1 shows the CONSORT study summary; Table 1, patient demographics; and Table 2, the hip arthroscopic findings and interventions performed on each patient.

A total of 10 patients were partially lost to follow up with missing VAS assessments over the $1.5-6$ hour postoperative study period (See Figure 1); 1 at all ward VAS time points; 4 at 2 hours; 4 at 4 hours; and 1 at 6 hours. Overall, patients' mean pain assessments appeared to be that of satisfactorily controlled pain; however recovery NRS-11 and postoperative VAS pain assessment ratings were highly variable (Tables 3 \& 4).

Pain assessments appeared to be of greatest magnitude in the recovery room and at the ward at 1.5 hours postoperatively [mean (SD) Recovery NRS-11 highest pain score 3.5 (3.0); Postoperative VAS 1.5 hrs $36 \mathrm{~mm}(18 \mathrm{~mm})$. Postoperative mean VAS approximately halved at the 6 hour postoperative time point. Figure 2 shows the highest, lowest and median recovery NRS-11; Figure 3, $1.5-6$ hour postoperative VAS. There was no statistically significant differences found between groups in pain assessments at any postoperative time points (See Tables $3 \& 4$ ). Correcting recovery NRS-11 and postoperative VAS for pre-operative VAS at rest did not change statistical significance except for the Lowest Recovery NRS-11 [ $\mathrm{p}=0.03$ between Group P (NRS-11 = 1.6) and Group L (NRS-11 = 0.7)]. Otherwise the largest difference between pain assessments was VAS $5 \mathrm{~mm}$ at the 1.5 and 2 hours postoperative time points.

$4 \%$ of patients received rescue FIB. Of the 6 patients receiving rescue FIB, all except 1 patient reported much improved pain; this patient was then prescribed a $0.15 \mathrm{mg} / \mathrm{kg} / \mathrm{hr}$ ketamine infusion.

Overall intraoperative \& recovery IV morphine equivalent analgesic doses were large [mean: SD (0.23: 0.1 $\mathrm{mg} / \mathrm{kg}$ ), with doses in the remainder of the 24 hour postoperative period much smaller (0.09: $0.1 \mathrm{mg} /$ $\mathrm{kg}$ ). There were no significant differences between groups in IV morphine equivalent analgesic doses (Group L = $0.22 \mathrm{mg} / \mathrm{kg}$; Group H = $0.23 \mathrm{mg} / \mathrm{kg}$; Group P = $0.24 \mathrm{mg} / \mathrm{kg}$ ). There were no significant differences in antiemetic doses between groups [largest difference between means in granisetron 
requirements $0.07 \mathrm{mg}(p=0.6)$; largest difference between means in cyclizine requirements $0.9 \mathrm{mg}(p=$ $0.9)]$. Figure 4 shows postoperative analgesic doses.

\section{Discussion}

We present findings from a double-blinded, 3-arm randomized controlled trial of 132 patients comparing the analgesic efficacy of 1) high dose IALA at operation end, 2) Iow dose IALA at operation end, and 3) IALA both pre-emptively and at operation end. With the exception of lowest recovery NRS-11 pain scores, the results of our primary and secondary outcome measures showed no statistically or clinically significant difference between IALA groups in recovery or postoperative pain score outcomes, morphine equivalent analgesic requirements, or antiemetic requirements. Our study was not powered to detect a significant difference in requirement for rescue FIB.

We believe the finding of a statistically significant difference in the preoperative NRS-11 corrected recovery lowest pain scores by one-way ANOVA and by bonferroni multiple comparisons testing between groups $L$ and $P$ to be spurious, as the variability in the low scores at this time point was artificially reduced by limiting the lowest possible corrected NRS-11 to zero. The mean NRS-11 difference here of 0.9 is also unlikely to be of clinical significance as the NRS-11 scores (Group P: 1.6 and Group L: 0.7 ) are both very low, correlating with established definitions of mild pain[11].

Whilst the use of IALA doses compared with placebo has shown promise in improving analgesia after hip arthroscopy, we specifically designed our study to compare variable dosing and timing of IALA. Baker et al found that $25 \mathrm{mg}$ postoperative IA bupivacaine had opioid sparing effects compared with the same dose infiltrated around portal insertion sites[7], and Morgenthaler et al used 50mg postoperative IA bupivacaine vs IA placebo to achieve a mean 20-hour VAS superiority of $10 \mathrm{~mm}$ at rest[6]. In knee arthroscopy, Tuncer et al[12] demonstrated superior analgesia up to 6 hours postoperatively when preemptive administration of $50 \mathrm{mg}$ IA bupivacaine was used prior to surgery, when compared with postoperative dosing. Goodwin demonstrated variable short-term pre-emptive effects[13, 14], whilst Fagan et al's study did not demonstrate superiority[15].

Several factors may account for the lack of any demonstrable effect with higher dose and pre-emptive administration of IALA in our study. Overall mean pain assessment scores were moderate-to-low, hence the perioperative use of good multimodal analgesia may have masked any benefit in IALA dosing or timing. Although we did not include a placebo arm in our study, we cannot exclude the possibility that any dose of IALA may lack significant efficacy when used in conjunction with systemic multimodal analgesia. Compared to our previous study[1] all patients received an arthroscopic fluid infusion pressure of 
$40 \mathrm{mmhg}$ rather than the more commonly used $80 \mathrm{mmhg}$, and fewer patients received femoral osteoectomy; factors known to predispose to postoperative pain. Overall opioid requirements were similar in both studies, however, and are approximately equivalent to the requirements observed in other hip arthroscopic studies[16]. We did not assess VAS on weight-bearing or movement of the hip joint postoperatively; the greater pain experienced under these circumstances may have demonstrated a preemptive or dose-dependent IALA effect. Morgenthaler's positive study findings included a larger effect size when assessment of pain on movement was used, however his team also demonstrated a statistically significant effect on pain at rest. IALA may have an effect on intra-articular sources of pain (eg. osteoectomy sites; labral repairs and their bony anchors) however will be unlikely to affect pain related to extra-articular causes (eg. peri-articular soft tissue distension from extravasated arthroscopic infusion fluid). Peri-articular LA infiltration appeared to be effective for postoperative pain in hip arthroscopy in one retrospective study[17].

The potential for arthroscopic fluid to have washed out the IALA before it has had the opportunity to block neural sodium channels is possible. This has not influenced the effect of postoperative hip arthroscopic IALA in previous studies but could have affected our pre-emptive dose; effective preoperative IALA used in some knee arthroscopic studies was given 20-30 minutes prior to portal insertion and irrigation[12].

Although our sample size was larger than most previous studies on IALA, the possibility of a type 2 error still exists. The further increase in statistical power afforded by our higher sample size however, adds strength to the existing literature that under a variety of surgical, patient and concomitant multimodal analgesic contexts, the analgesic effect of IALA is unlikely to be clinically significant. This increase in statistical power was achieved by intentionally reducing the VAS difference initially sought. This VAS difference may be considered clinically insignificant, but permits a larger sample size calculation for our study. Our power calculation was defined by VAS improvements at rest on a dose-to-VAS $\mathrm{mm}$ effect, however the comparator patient population in the study from where this data was obtained were all fishermen[6], who may have had a higher proportion of severe underlying osteoarthritis or need for femoral osteoectomy. This may have relatively amplified the efficacy of IALA in this study. As our study lacks a placebo arm, no comparative inferences can be made from our results regarding the effect of any local anaesthetic regime against no local anaesthetic at all when administered simultaneously with multimodal systemic analgesia. Our use of NRS-11 in the recovery room and VAS in the postoperative ward environment limits absolute comparisons between the pain assessments in the two scenarios. Whilst the two correlate, there is not complete concordance between these measures of pain severity.

\section{Conclusions}


Use of $100 \mathrm{mg}$ vs $200 \mathrm{mg}$ IALA at operation end, or $100 \mathrm{mg}$ pre-emptively and at operation end, appears to have no differing effects on acute pain, analgesic requirements, or antiemetic requirements after hip arthroscopy. Pain after therapeutic hip arthroscopy is highest in the recovery room and first 1.5 hours postoperatively, improving significantly by 6 hours postoperatively, and can be well managed with multimodal analgesia and appropriately titrated opioid administration. Rescue fascia iliaca blockade may be required in a small proportion of patients. As lower doses of IA ropivacaine do not appear to offer inferior analgesia in this context, we recommend that if IALA is to be used at all, use of $100 \mathrm{mg}$ IA ropivacaine will result in a lower total dose of LA administered to patients if rescue FIB of a given dose is required.

\section{Abbreviations}




\begin{tabular}{|c|c|}
\hline Abrreviation & Definition \\
\hline ALOHA & Intra-Articular IOcal anaesthetic in Hip Arthroscopy \\
\hline NRS-11 & 11-point Numerical Rating Score for pain \\
\hline LA & Local anaesthetic \\
\hline IALA & Intra articular local anaesthetic \\
\hline SD & Standard deviation \\
\hline$p=$ & P-value \\
\hline VAS & Visual Analogue Scale for pain \\
\hline FIB & Fascia Iliaca Block \\
\hline ACTRN & Australian \& New Zealand Clinical Trials Register \\
\hline HREC & Human Research \& Ethics Committee \\
\hline CONSORT & Consolidated Standards of Reporting Trials \\
\hline IV & intravenous \\
\hline $\mathrm{mg}$ & milligram \\
\hline $\mathrm{mcg}$ & microgram \\
\hline PCA & Patient controlled analgesia \\
\hline bd & Twice daily \\
\hline tds & Three times daily \\
\hline ANOVA & Analysis of variance \\
\hline $\mathrm{mm}$ & millimetres \\
\hline \# & number \\
\hline$\%$ & percent \\
\hline ASA & American Society of Anaesthesiologists score \\
\hline mins & minutes \\
\hline $\mathrm{Kg}$ & kilogram \\
\hline $\mathrm{Hr}$ & hour \\
\hline
\end{tabular}

\section{Declarations}




\section{- Ethics approval and consent to participate}

This study was approved by our institution's Human Research and Ethics committee (Melbourne Health HREC, approval \# 2013.195). Approval was granted for protocol variation when increased sample size was necessary to avoid a Type 2 error in the outcome measures (\#2013.195.P1-2). All patients provided written informed consent to be included in the trial.

\section{- Consent for publication}

Not Applicable

\section{- Availability of data and material}

The datasets used and/or analysed during the current study are available from the corresponding author on reasonable request. Restrictions apply to the availability of these data, which were used under license for the current study, and so are not publicly available. Data are however available from the authors upon reasonable request and with permission of Bellbird Private Hospital administration

\section{- Competing interests}

The authors declare that they have no competing interests.

\section{- Funding}

No external funding was sourced for the design of the study; the collection, analysis, and interpretation of data; and in the writing of the manuscript.

\section{- Authors' contributions}

CT made substantial contributions to the conception and design of the study, the acquisition of data, and the analysis/ interpretation of data. He also drafted and revised the manuscipt, and approved the final version for publication.

PT made substantial contributions to the conception and design of the study, the acquisition of data, and the analysis/ interpretation of data. He also drafted and revised the manuscipt, and approved the final version for publication.

YMC made substantial contributions to the conception of the study, revised the manuscipt for publication, and approved the final version for publication. 
WH made substantial contributions to the conception and design of the stud. He also drafted and revised the manuscipt, and approved the final version for publication.

LW made substantial contributions to the conception and design of the study and the analysis/ interpretation of data. He also drafted and revised the manuscipt, and approved the final version for publication.

All authors read and approved the final manuscript.

- Acknowledgements

Not Applicable

- Authors' information

Chong 0. Tan, MBBS, FANZCA, M (Clinical Ultrasound)

Position: Staff Anesthetist

Department: Anaesthesia

Institution: Austin Hospital, Level 2 Austin Towers, 145 Studley Rd Heidelberg,

VIC 3084, Melbourne, AUSTRALIA

\section{PhongTan, MBBS (Hons), FRACS (Ortho)}

Position: Director of Orthopaedics

Department: Surgery

Institution: Western Hospital, 60 Gordon St, Footscray VIC 3011, Melbourne, AUSTRALIA

\section{YewMing Chong, MBBS}

Position: Registrar

Department: Surgery 
Institution: Western Hospital, 60 Gordon St, Footscray VIC 3011, Melbourne, AUSTRALIA

\section{William Howard,_MBBS, FANZCA, FFPMANZCA}

Position: Director of Pain Services

Department: Anaesthesia, Pain and Perioperative Medicine

Institution: Austin Hospital, Level 2 Austin Towers, 145 Studley Rd Heidelberg,

VIC 3084, Melbourne, AUSTRALIA

\section{Laurence Weinberg,_BSc, MBBCh, MRCP, DipCritCareEcho, FANZCA,MD}

Position: Director

Department: Anaesthesia, Pain and Perioperative Medicine

Institution: Austin Hospital, Level 2 Austin Towers, 145 Studley Rd Heidelberg, VIC 3084, Melbourne, AUSTRALIA

\section{References}

1. Tan CO, Chong YM, Tran P, Weinberg L, Howard W. Surgical predictors of acute postoperative pain after hip arthroscopy. BMC Anesthesiol. 2015;15:96.

2. YaDeau JT, Tedore T, Goytizolo EA, Kim DH, Green DST, Westrick A, et al. Lumbar plexus blockade reduces pain after hip arthroscopy: a prospective randomized controlled trial. Anesth Analg. 2012;115:968-72.

3. Schroeder KM, Donnelly MJ, Anderson BM, Ford MP, Keene JS. The analgesic impact of preoperative lumbar plexus blocks for hip arthroscopy. A retrospective review. Hip Int J Clin Exp Res Hip Pathol Ther. 2013;23:93-8.

4. Nye ZB, Horn J-L, Crittenden W, Abrahams MS, Aziz MF. Ambulatory continuous posterior lumbar plexus blocks following hip arthroscopy: a review of 213 cases. J Clin Anesth. 2013;25:268-74.

5. Krych AJ, Baran S, Kuzma SA, Smith HM, Johnson RL, Levy BA. Utility of multimodal analgesia with fascia iliaca blockade for acute pain management following hip arthroscopy. Knee Surg Sports Traumatol Arthrosc Off J ESSKA. 2014;22:843-7. 
6. Morgenthaler K, Bauer C, Ziegeler S, Mencke T, Werth M, Seil R, et al. [Intra-articular bupivacaine following hip joint arthroscopy. Effect on postoperative pain]. Anaesthesist. 2007;56:1128-32.

7. Baker JF, McGuire CM, Byrne DP, Hunter K, Eustace N, Mulhall KJ. Analgesic control after hip arthroscopy: a randomised, double-blinded trial comparing portal with intra-articular infiltration of bupivacaine. Hip Int J Clin Exp Res Hip Pathol Ther. 2011;21:373-7.

8. Ong CK-S, Lirk P, Seymour RA, Jenkins BJ. The Efficacy of Preemptive Analgesia for Acute Postoperative Pain Management: A Meta-Analysis. Anesth Analg. 2005;100:757-73.

9. Dalens B, Vanneuville G, Tanguy A. Comparison of the fascia iliaca compartment block with the 3-in1 block in children. Anesth Analg. 1989;69:705-13.

10. Ooi K. Faculty of Pain Medicine | ANZCA: Official Opioid Dose Equivalence. FPMANZCA Prof Doc. 2014. http://www.anzca.edu.au/documents/anzcafpm-good-prescribing-practice-20150814.pdf. Accessed 14 Apr 2017.

11. Gerbershagen HJ, Rothaug J, Kalkman CJ, Meissner W. Determination of moderate-to-severe postoperative pain on the numeric rating scale: a cut-off point analysis applying four different methods. Br J Anaesth. 2011;107:619-26.

12. Tuncer $B$, Babacan $C A$, Arslan M. The pre-emptive analgesic effect of intra-articular bupivacaine in arthroscopic knee surgery. Acta Anaesthesiol Scand. 2005;49:1373-7.

13. Goodwin RC, Parker RD. Comparison of the analgesic effects of intra-articular injections administered preoperatively and postoperatively in knee arthroscopy. J Knee Surg. 2005;18:17-24.

14. Goodwin RC, Amjadi F, Parker RD. Short-term analgesic effects of intra-articular injections after knee arthroscopy. Arthrosc J Arthrosc Relat Surg Off Publ Arthrosc Assoc N Am Int Arthrosc Assoc. 2005;21:307-12.

15. Fagan DJ, Martin W, Smith A. A randomized, double-blind trial of pre-emptive local anesthesia in daycase knee arthroscopy. Arthrosc J Arthrosc Relat Surg Off Publ Arthrosc Assoc N Am Int Arthrosc Assoc. 2003;19:50-3.

16. Baker JF, Byrne DP, Hunter K, Mulhall KJ. Post-operative opiate requirements after hip arthroscopy. Knee Surg Sports Traumatol Arthrosc. 2011;19:1399-402.

17. Philippi MT, Kahn TL, Adeyemi TF, Maak TG, Aoki SK. Extracapsular local infiltration analgesia in hip arthroscopy: a retrospective study. J Hip Preserv Surg. 2018;5:60-5.

\section{Tables}

TABLE 1 - PATIENT DEMOGRAPHICS, SURGICAL DURATION \& NEED FOR RESCUE REGIONAL ANALGESIA 


\begin{tabular}{|l|l|l|l|}
\hline & Group L & Group H & Group P \\
\hline Age (mean: SD) & $40(12)$ & $38(11)$ & $38(11)$ \\
\hline Male (\#:\% ) & $54(37 \%)$ & $84(58 \%)$ & $86(60 \%)$ \\
\hline Weight (Kg: mean [SD]) & $78(16)$ & $77(15)$ & $78(20)$ \\
\hline \# ASA 3 & & & \\
\hline Surgical Duration [mins(mean:SD)] & $30(17)$ & $31(16)$ & $28(15)$ \\
\hline Rescue FIB required (\# patients) & 3 & 1 & 2 \\
\hline & 2 & 3 & 5 \\
\hline & & & \\
\hline
\end{tabular}

a: all remaining patients were ASA 1 and 2.

$b:$ FIB $=$ fascia iliaca block

TABLE 2 - ARTHROSCOPIC FINDINGS \& SURGICAL INTERVENTIONS PERFORMED 


\begin{tabular}{|c|c|c|c|}
\hline & Group L $(n=44)$ & Group H $(n=44)$ & Group P $(n=44)$ \\
\hline Femoral Osteoectomy & 10 & 15 & 11 \\
\hline Acetabular Osteoectomy & 4 & 12 & 7 \\
\hline Labral repair & 24 & 34 & 34 \\
\hline Rim Chondroplasty & 23 & 30 & 32 \\
\hline Microfracture & 2 & 0 & 3 \\
\hline Ligamentum Teres debridement & 35 & 38 & 38 \\
\hline Capsular Repair & 1 & 1 & 0 \\
\hline Acetabular Chondral Damage & 28 & 34 & 36 \\
\hline Extra-articular Approach & 2 & 2 & 0 \\
\hline Intra-Articular Steroid Used & 14 & 5 & 6 \\
\hline
\end{tabular}




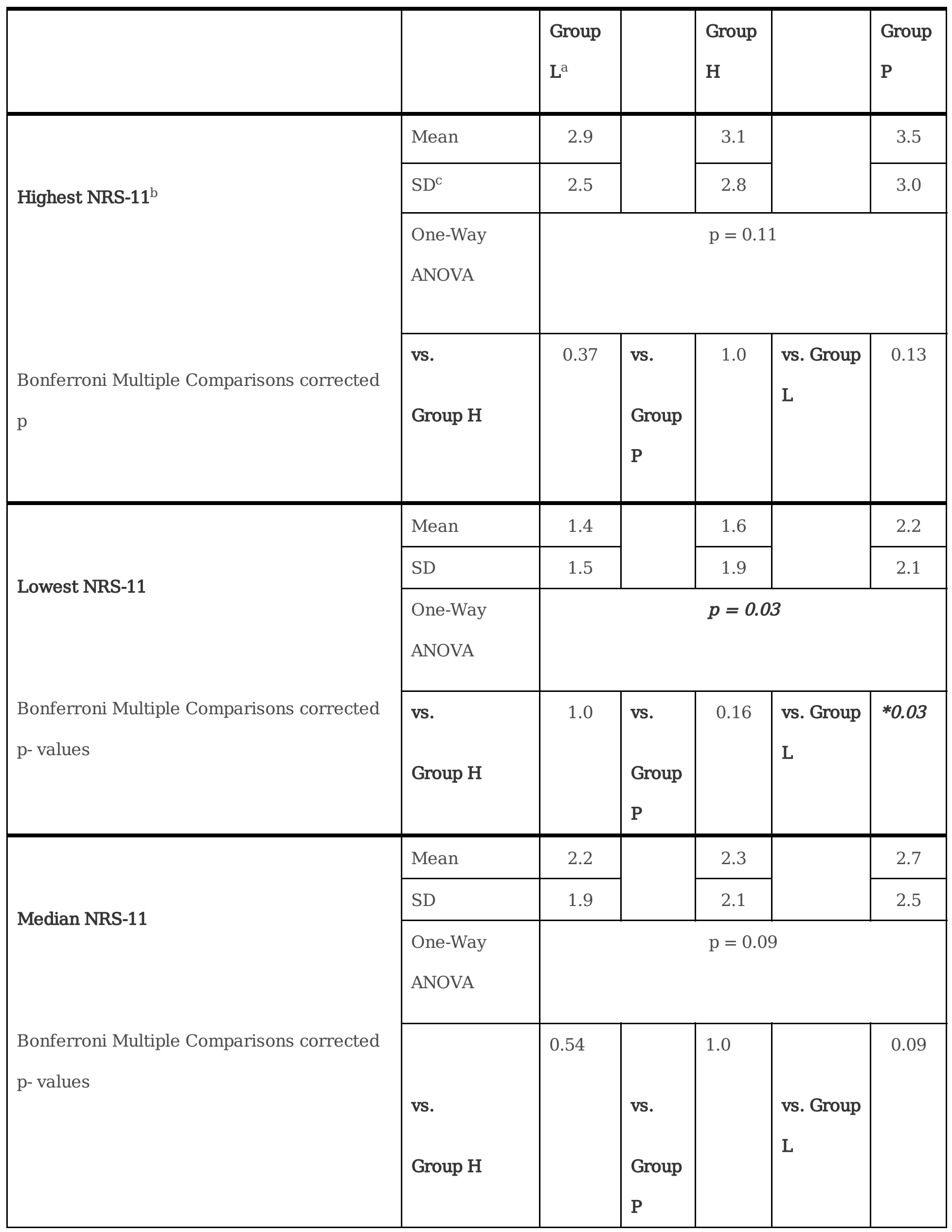


a: Group L = Low dose ropivacaine group, 100mg IALA at operation end; Group H = High dose ropivacaine group, 200mg IALA at operation end; Group P= Pre-emptive ropivacaine group, 100mg ropivacaine prior to operation start and at operation end.

b: 11-point pain Numerical Rating Scale

C: Standard deviation

TABLE 4 - POSTOPERATIVE 11-POINT NUMERICAL RATING SCALE ASSESSMENTS OF PAIN 


\begin{tabular}{|c|c|c|c|c|c|c|}
\hline & & $\begin{array}{l}\text { Group } \\
\mathrm{L}^{\mathrm{a}}\end{array}$ & & $\begin{array}{l}\text { Group } \\
\text { H }\end{array}$ & & $\begin{array}{l}\text { Group } \\
\text { P }\end{array}$ \\
\hline \multirow{3}{*}{$\mathrm{VAS}^{\mathrm{b}} 1.5 \mathrm{hrs}(\mathrm{mm})$} & Mean & 33 & & 31 & & 36 \\
\hline & $\mathrm{SD}^{\mathrm{C}}$ & 18 & & 16 & & 19 \\
\hline & $\begin{array}{l}\text { One-Way } \\
\text { ANOVA }\end{array}$ & \multicolumn{5}{|c|}{$p=0.33$} \\
\hline $\begin{array}{l}\text { Bonferroni Multiple Comparisons corrected } \\
\text { p }\end{array}$ & $\begin{array}{l}\text { vs. } \\
\text { Group H }\end{array}$ & 1.0 & $\begin{array}{l}\text { vs. } \\
\text { Group } \\
\text { P }\end{array}$ & 0.93 & $\begin{array}{l}\text { vs. Group } \\
\text { L }\end{array}$ & 0.44 \\
\hline VAS $2 \mathrm{hrs} \mathrm{(mm)}$ & Mean & 29 & & 26 & & 31 \\
\hline \multirow{3}{*}{ Bonferroni Multiple Comparisons corrected } & SD & 14 & & 17 & & 17 \\
\hline & $\begin{array}{l}\text { One-Way } \\
\text { ANOVA }\end{array}$ & \multicolumn{5}{|c|}{$\mathrm{p}=0.59$} \\
\hline & $\begin{array}{l}\text { vs. } \\
\text { Group H }\end{array}$ & 1.0 & $\begin{array}{l}\text { vs. } \\
\text { Group } \\
\text { P }\end{array}$ & 1.0 & $\begin{array}{l}\text { vs. Group } \\
\text { L }\end{array}$ & 0.92 \\
\hline \multirow{3}{*}{ VAS $4 \mathrm{hrs}$} & Mean & 20 & & 20 & & 22 \\
\hline & $\mathrm{SD}$ & 14 & & 13 & & 18 \\
\hline & $\begin{array}{l}\text { One-Way } \\
\text { ANOVA }\end{array}$ & \multicolumn{5}{|c|}{$\mathrm{p}=0.28$} \\
\hline $\begin{array}{l}\text { Bonferroni Multiple Comparisons corrected } \\
\text { p-values }\end{array}$ & $\begin{array}{l}\text { vs. } \\
\text { Group H }\end{array}$ & 0.37 & $\begin{array}{l}\text { vs. } \\
\text { Group } \\
\text { P }\end{array}$ & 1.0 & $\begin{array}{l}\text { vs. Group } \\
\text { L }\end{array}$ & 0.6 \\
\hline
\end{tabular}


a: Group L = Low dose ropivacaine group, 100mg IALA at operation end; Group H = High dose ropivacaine group, 200mg IALA at operation end; Group P= Pre-emptive ropivacaine group, 100mg ropivacaine prior to operation start and at operation end.

$b$ : Visual Analogue Scale

c: Standard deviation

\section{Figures}

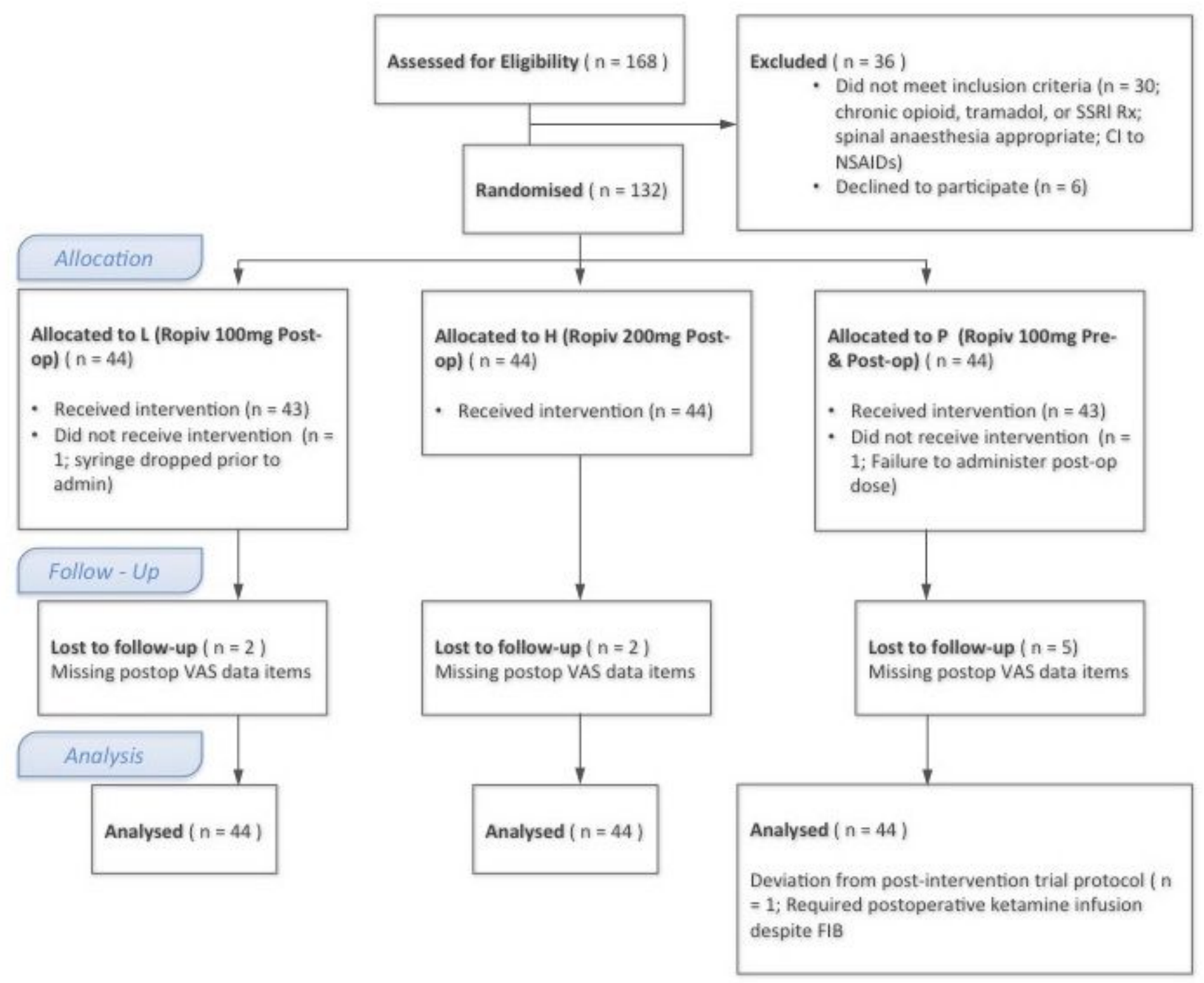


Figure 1

ALOHA CONSORT DIAGRAM

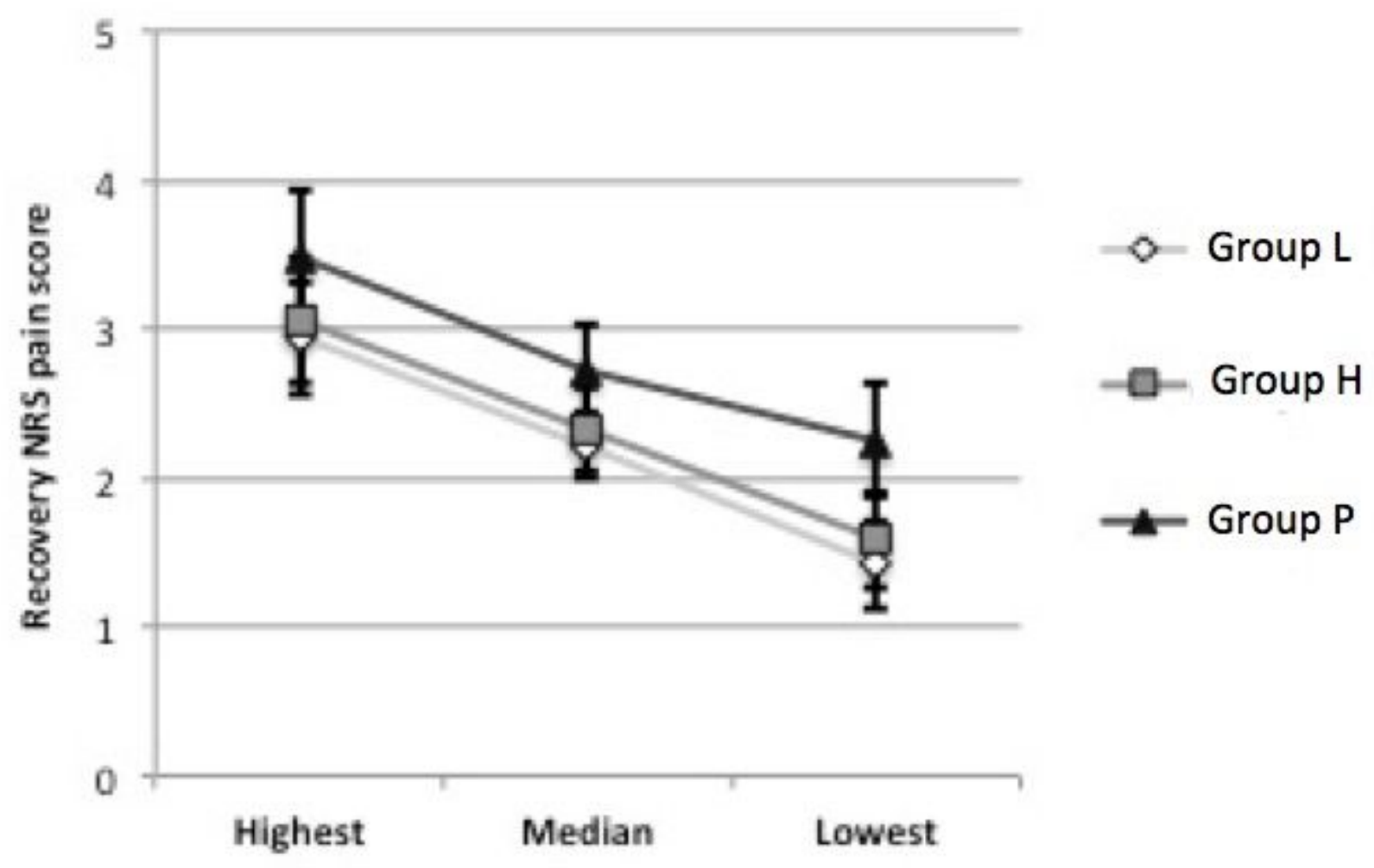

a. Error bars are 1 SEM.

Figure 2

RECOVERY NRS-11 PAIN SCORES 


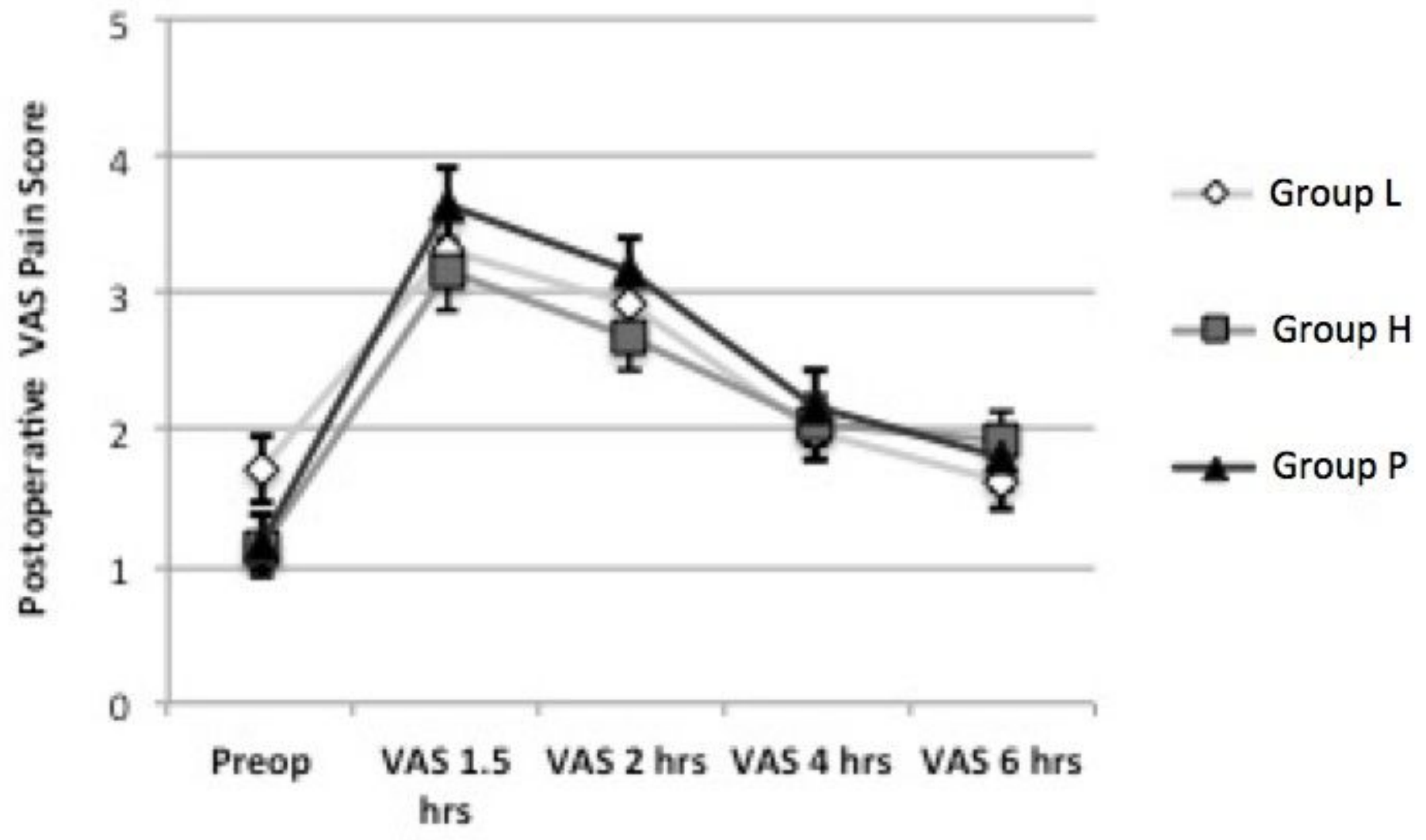

a. Error bars are 1 SEM.

Figure 3

POSTOPERATIVE VAS PAIN SCORES 


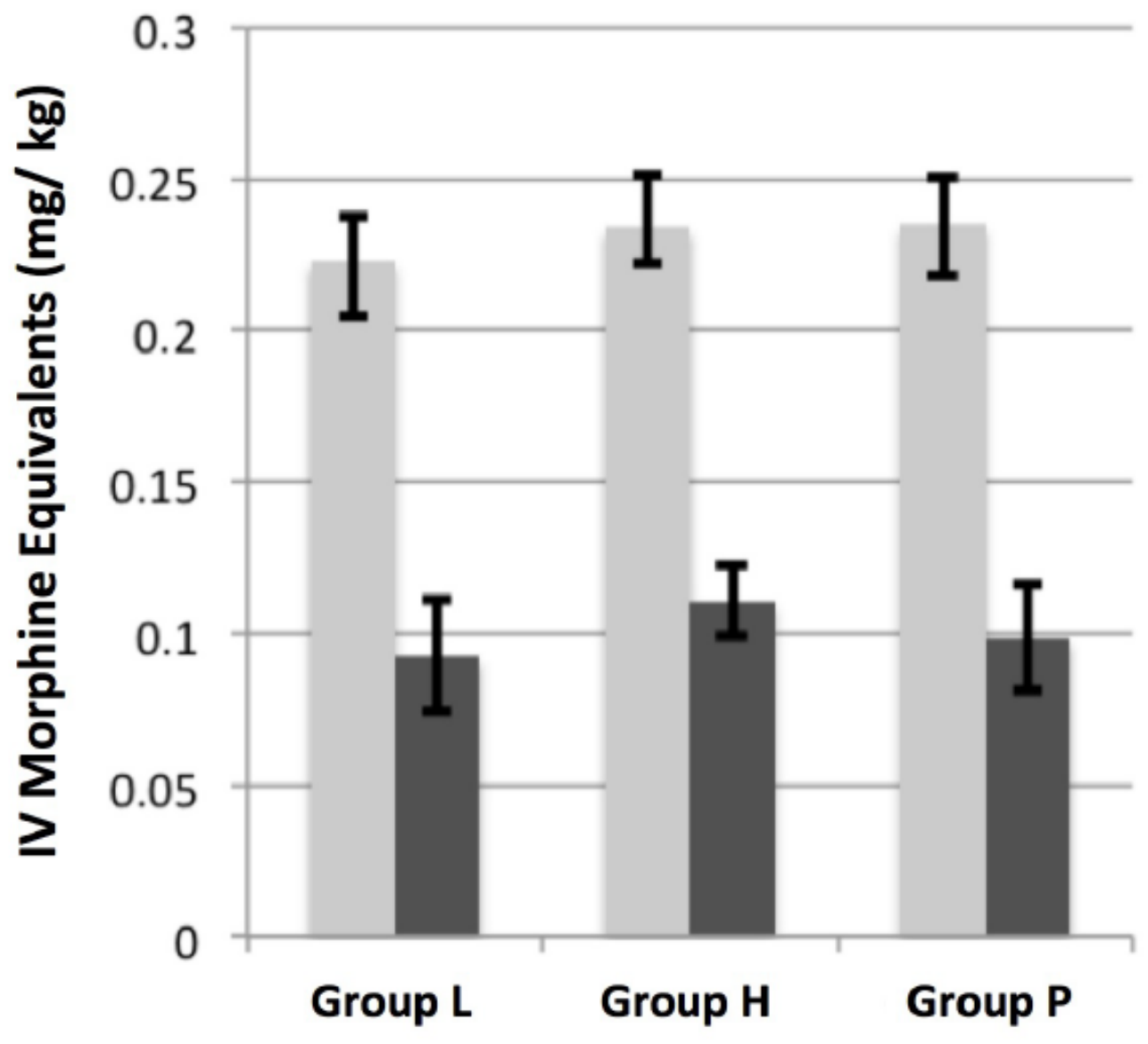

Figure 4

24 HOUR POSTOPERATIVE IV MORPHINE EQUIVALENT REQUIREMENTS
Intraoperative \& Recovery Opioid Requirements

$1^{\text {st }} 24$ hours Ward Opioid Requirements 